\title{
Unexpected influence of substituents on the binding affinities of polycyclic aromatic hydrocarbons with a tetra-Au(I) metallorectangle
}

\author{
Susana Ibáñez, ${ }^{a}$ Dmitry G. Gusev ${ }^{\mathrm{b}}$ and Eduardo Peris ${ }^{\mathrm{a} *}$ \\ a Institute of Advanced Materials (INAM). Centro de Innovación en Química Avanzada (ORFEO-CINQA). \\ Universitat Jaume I. Av. Vicente Sos Baynat s/n. Castellón. E-12071. Spain. Email: eperis@uji.es \\ ${ }^{\mathrm{b}}$ Dept. of Chemistry and Biochemistry, Wilfrid Laurier University, Waterloo, Ontario N2L 3C5 (Canada).
}

\begin{abstract}
A tetra-gold supramolecular organometallic cage constructed with two pyrene-bis-imidazolylidene ligands and two carbazolyl-bis-alkynyl linkers (1) was studied as host for a series of substituted polycyclic aromatic hydrocarbons (PAHs). For the two smaller PAHs used (2-naphthalenol and 1-pyrenemethanol), the presence of the $\mathrm{OH}$ groups at the periphery of the molecules did not enhance the binding affinity of the guest, compared with the unsubstituted PAHs. This observation indicated no hydrogen bonding of these two guests with the $\mathrm{NH}$ of the carbazole linker, as well as negligible dispersive interactions of the substituents with the $\pi$-system of 1 . In the case of 3 perylenemethanol, the $\mathrm{CH}_{2} \mathrm{OH}$ group produced a significant increase in the binding affinity, vs perylene. Similarly, 3methylperylene shows an increased binding affinity compared to perylene. MN15-L/def2-QZVP calculations gave Gibbs reaction energies for the displacement of perylene from the host by the substituted perylenes becoming more exergonic in the order: -1.6 (3-methylperylene) > -4.3 (3-ethylperylene) > -4.5 kcal/mol (3-perylenemethanol). The experimental and DFT results indicate that the peripheral dispersive interactions can make a significant contribution to the host-guest bonding energy, in addition to the conventional $\pi-\pi$-stacking interactions. Our work highlights the importance of dispersive interactions in the contribution to the binding affinity of host-guest chemistry complexes. .
\end{abstract}

\section{INTRODUCTION}

Polycyclic aromatic hydrocarbons (PAHs) are naturally occurring compounds that are widespread environmental contaminants. ${ }^{1}$ Lower PAHs (2-3 rings) are known to exhibit acute toxicity, while most of the larger PAHs (4to 7-rings) are carcinogenic to a variety of organisms. On the other hand, the rigid planar conjugated structures of PAHs endow unique optical and morphological properties that have found multiple applications in the fabrication of optoelectronic devices, ${ }^{2}$ such as light emitting diodes (OLEDs) ${ }^{3}$ and organic field-effect transistors (OFETs). ${ }^{4}$ The properties of a $\mathrm{PAH}$ are strongly dependent on its structure, which is mostly determined by the number of aromatic rings, the manner in which the rings are fused, and the nature of their substituents. In addition, the introduction of functionalities at the periphery of the $\mathrm{PAH}$ can allow tuning its properties, so that their utility in the fabrication of tailor-made materials and devices can be expanded. ${ }^{5}$ The problems PAHs cause as pollutants, together with their high value for the fabrication of optoelectronic devices, have motivated the development of a large number of molecular hosts for selective $\mathrm{PAH}$ encapsulation and sequestration. ${ }^{6}$

NHCs (N-heterocyclic carbenes) are currently recognized as very convenient scaffolds for the construction of Supramolecular Organometallic
Complexes (SOCs). ${ }^{7}$ During the last four years we described a series of NHC-based supramolecular organometallic complexes (SOCs), ${ }^{8}$ among which several were used as hosts for the encapsulation of PAHs. ${ }^{9}$ In particular, we recently described a tetra-gold metallorectangle that combines two pyrene-bisimidazolylidene ligands with two carbazolyl-bis-alkynyl linkers (1, in Scheme 1). ${ }^{9 a}$ The two cofacial pyrene moieties separated by almost exactly $7 \AA$, render this molecule a very effective receptor toward $\mathrm{PAH}$ molecules, and the observed binding affinities were among the largest reported for a metallosupramolecular host. $^{9 a}$ The recognition abilities of the metallosupramolecular host 1 are explained by the effective $\pi-\pi$ stacking interactions between the host and the $\mathrm{PAH}$ guest. Incorporation of functionalities in the host
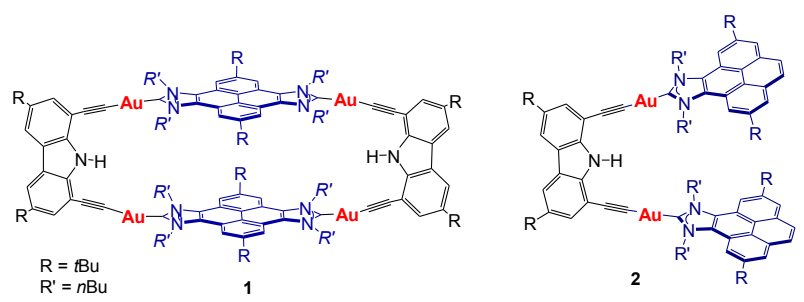

that add further non-covalent interactions with the $\mathrm{PAH}$ molecules can provide methods for enhancing guest selectivity and facilitate the separation of structurally similar organic molecules. Recently, Li and Stoddart 
highlighted the importance of lateral interactions for designing hosts capable of binding guests in multiple locations and modes. ff $^{\mathrm{We}}$ also showed that for a pyrene-Au(I)-based metallotweezer with a carbazolyl linker (2, in Scheme 1), the presence of the $\mathrm{N}-\mathrm{H}$ group in the carbazole linker enabled the receptor to show significant enhanced binding toward PAHs substituted with $\mathrm{H}$-bonding groups, as a consequence of the combined action of $\pi-\pi$ stacking and hydrogenbonding. ${ }^{9 e}$

Scheme 1. Carbazolyl-connected tetra-Au(I) metallorectangle (1) and di-Au(I) metallotweezer (2).

With these precedents in hand, we now present our studies on how the presence of substituents on $\mathrm{PAH}$ molecules influence their binding affinities with our tetra$\mathrm{Au}(\mathrm{I})$ metallorectangle 1. As will be described in the following sections, the binding affinities of these guests are highly influenced by dispersive London forces, a relatively weak interaction that has been largely ignored in discussions of molecular stability and reactivity, ${ }^{10}$ and for interpreting the formation of host-guest inclusion complexes. ${ }^{11}$

\section{RESULTS AND DISCUSSION}

In order to determine whether the presence of substituents capable of hydrogen bonding would influence the binding affinities of PAHs with the metallorectangle $\mathbf{1}$, four substituted polycyclic aromatic hydrocarbons depicted in Scheme 2 were used as guests to form the corresponding inclusion complexes with 1. Compounds 3-5 contain an $\mathrm{OH}$ group, which, in principle, can form a hydrogen bond with an $\mathrm{NH}$ group of 1. For comparison, for the reasons that will be fully explained below, we also included 3-metylperylene (6) in this study. 3-Ethylperylene (7) is included in Scheme 2

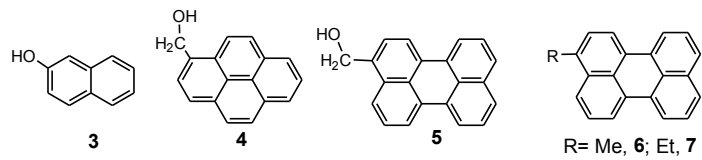

because it was used as a model guest in our DFT studies (vide infra).

Scheme 2. Substituted PAHs used in the study.

For the determination of the binding affinities with $\mathbf{3}-\mathbf{6}$, we performed ${ }^{1} \mathrm{H}$ NMR titrations using the same conditions as we used before for the determination of the association constants of 1 with the corresponding unfuctionalized PAHs. ${ }^{9 a}$ Hence, the titrations were carried out in $\mathrm{CD}_{2} \mathrm{Cl}_{2}$, at room temperature, using a constant concentration of $\mathbf{1}$. In all cases, addition of the substituted PAHs induced an upfield ${ }^{1} \mathrm{H}$ NMR shift of the pyrene protons of 1 . Together with these changes, we also observed that the signal due to the $\mathrm{NH}$ proton shifted downfield upon addition of the guests (see ESI for details). This shift was significantly larger with $\mathbf{4}$ and $\mathbf{5}$, compared to the smaller functionalized $\mathrm{PAH}, \mathbf{3}$. In this regard, with 2-naphthalenol (3), the upfield shift of the signal due to the $\mathrm{NH}$ proton was practically negligible, while the largest shift observed during the titrations with 1-pyrene-methanol (4) and 3-perylenemethanol (5) was $+0.75 \mathrm{ppm}$ in both cases. This large upfield shift observed for the signal of the $\mathrm{NH}$ proton in 1 upon addition of $\mathbf{4}$ and $\mathbf{5}$ contrasts with the smaller shifts observed when the related unsubstituted PAHs were added, which were 0.2 and $0.4 \mathrm{ppm}$, for pyrene and perylene, respectively, as we previously reported.9a Based on the changes observed, we were able to determine the association constants of 1 with the smaller substituted PAHs $\mathbf{3}$ and $\mathbf{4}$, by processing the data using a 1:1 stoichiometric model. ${ }^{12}$ The association constants found were $20 \pm 1$ and $3360 \pm 90 \mathrm{M}^{-1}$, for 3 and $\mathbf{4}$, respectively, i.e. almost identical to those found previously for naphthalene and pyrene..$^{9 a}$ These values indicate that for the case of 2-naphthalenol and 1pyrenemethanol, the presence of the hydroxyl group does not provide any enhancement in the binding affinity of the guest, compared to the unsubstituted analogue $\mathrm{PAH}$ molecules. This result may be due to the location of the functionalized guest molecule in the cavity of the guest, in a manner where a maximum face-to-face overlap with the pyrene panels of the host is established, in an orientation where the $\pi-\pi$-stacking interaction between the host and the guest is maximized. We think that, in this situation, the establishment of an effective hydrogen bonding interaction with the $\mathrm{NH}$ group of the carbazolyl linker might require the guest molecule to shift from the position that facilitates the maximum $\pi-\pi$ stacking interaction. In addition, the establishment of a hydrogen bond between the guest and the $\mathrm{NH}$ group of the host should require that the guest accommodates in such a manner that needs to fit between the two gold atoms in order to come close enough to the $\mathrm{NH}$ group. With the two Au atoms separated by a distance of about $6.5 \AA$ in the empty molecule of $\mathbf{1}$, the approach of the guest to the $\mathrm{NH}$ bond should be accompanied by metal-guest interactions (vide infra). This situation contrasts with the easy approach of guests to the $\mathrm{NH}$ bond in the metallotweezer $\mathbf{2}$, as we demonstrated earlier. ${ }^{9 e}$ In this case, the molecule is flexible enough as for allowing that the two gold atoms separate and accommodate to show the most favorable orientation with respect to the molecule of guest upon hydrogen bond formation with the $\mathrm{NH}$ group of the carbazole linker.

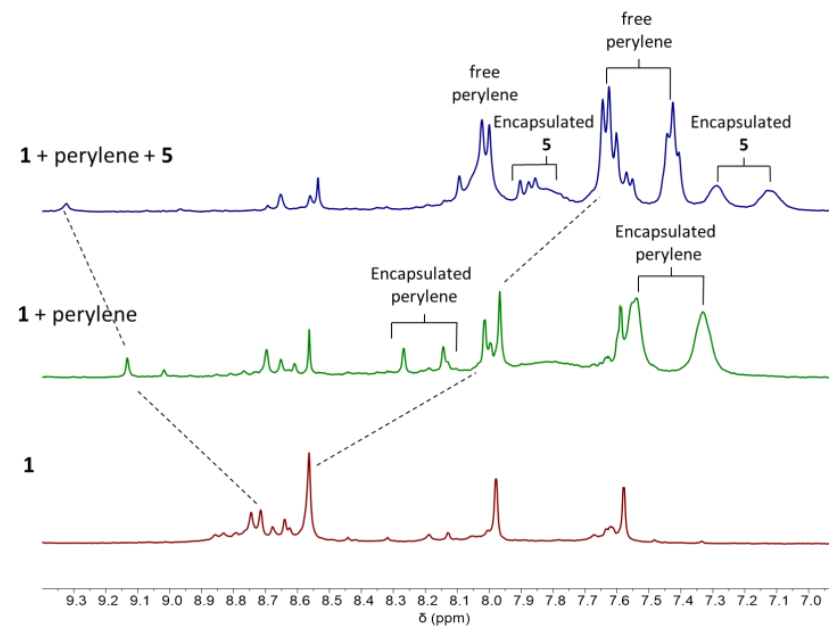

Figure 1. Selected region of the ${ }^{1} \mathrm{H}$ NMR spectra $\left(\mathrm{CD}_{2} \mathrm{Cl}_{2}\right)$ of 1 , and subsequent addition of one equivalent of perylene (middle), and 5 (top). The shifts observed for two representative signals of the host are also indicated. 
The NMR spectra resulting from the titrations performed with 3-perylenemethanol could not be used for determining its association constant, because the value apparently exceeds the limits for being determined by NMR spectroscopic means (NMR titrations are only valid for determining constants below $\left.10^{4}-10^{5} \quad M^{-1}\right) .{ }^{12}$ Unfortunately, the titrations performed using Uv-vis or fluorescence spectroscopy did not provide reliable results that could give us a meaningful association constant. Neither the Uv-vis nor the fluorescence titrations showed distinctive bands that could be used to monitor the consumption of the free guest and formation of the host-guest adduct upon addition of increasing amounts of $\mathbf{5}$. Under these circumstances, we decided to perform an experiment in order to assess whether the association constant of $\mathbf{5}$ was larger than that previously found for perylene $\left(1.6 \times 10^{5} \mathrm{M}^{-1}\right) \cdot{ }^{9 a}$ The experiment involved preparing a solution of perylene@1 by adding one equivalent of perylene to 1 , followed by addition of a stoichiometric amount of $\mathbf{5}$ in order to see if $\mathbf{5}$ replaces perylene in the cavity of the host. As can be observed from the series of spectra shown in Figure 1, the addition of one equivalent of perylene to a solution of 1 instantly produces the quantitative formation of perylene@1. Subsequent addition of one equivalent of $\mathbf{5}$, resulted in the complete encapsulation of 5 to form $5 @ 1$, with the concomitant release of perylene. This simple experiment demonstrates that the binding constant associated with the formation of $5 @ 1$ is significantly larger than that for perylene@1.

Single crystals suitable for X-ray diffraction studies of 5@1, were obtained by slow diffusion of hexane into a concentrated solution of the complex in chloroform. The molecular structure of this host-guest complex is shown in Figure 2. The structure of $5 @ 1$ confirms that a molecule of 3-perylenemethanol is accommodated inside the cavity of the metallorectangle 1 . The host structure has a two-fold symmetry axis that connects the two centroids of the pyrene moieties of the metallorectangle, therefore the $-\mathrm{CH}_{2} \mathrm{OH}$ functionality of the guest occupies two symmetry-related orientations in the molecule, with $50 \%$ occupancy each one (only one of these orientations is shown in Figure 2). The distance between the planes formed by the cofacial pyrene units is $7.142 \AA$, therefore very similar to the one shown for the empty host $(7.214$ $\AA$ ), ${ }^{9 a}$ indicating that the encapsulation of the guest occurs at a minimum energy cost due to the negligible inducedfit distortions. It is important to point out that the centroid of the planar guest is perfectly aligned with the centroids of the pyrene panels of the host. In addition, the longer axis of the perylene moiety of $\mathbf{5}$ is perfectly aligned with the longer axis of the pyrene-di-imidazolylidene ligands of the metallorectangle, therefore rendering an orientation for which the face-to-face contact between the host and the guest is maximized. This orientation of the guest within the cage is identical to that found for the structure of perylene@1, ${ }^{\text {a }}$ therefore indicating that the presence of the $-\mathrm{CH}_{2} \mathrm{OH}$ functionality has negligible influence on the orientation of the guest within the cavity of the metallorectangle. The distance between the oxygen atom of the guest and the $\mathrm{NH}$ hydrogen atom of the carbazolyl linker of the host is $5.58 \AA$, therefore the existence of a hydrogen bonding interaction can be fully discarded (at least for the solid-state structure of the complex).

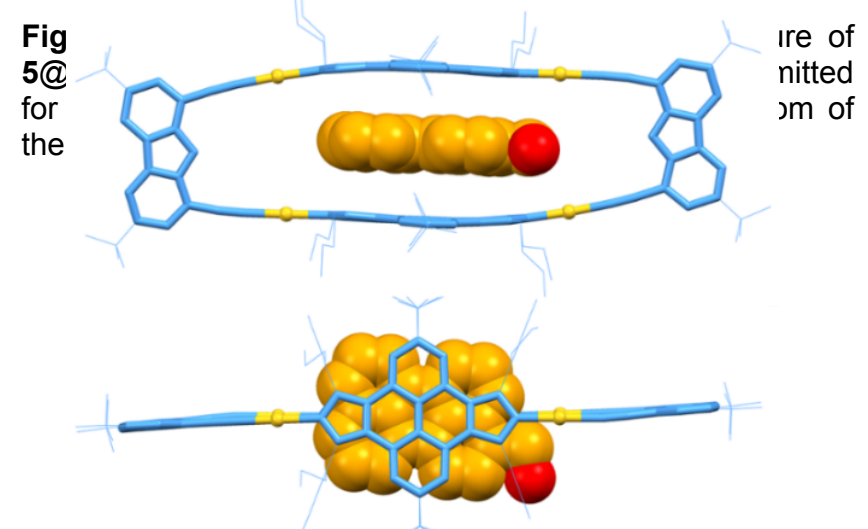

The experimental results described above suggest that the presence of the hydroxyl groups in $\mathbf{3}$ and $\mathbf{4}$ do not provide any measurable enhancement in the binding affinities of these guests, compared to their unfunctionalized analogues. For the case of $\mathbf{5}$, the experiments show that the binding affinity of this guest is significantly enhanced compared to that found for perylene. However, as observed from the X-ray diffraction molecular structure of 5@1, this enhancement cannot be ascribed to a hydrogen bonding interaction in the resulting host-guest complex. To shed some light on this point, we decided to study the binding affinity of 3methylperylene (6) with our metallorectangle 1. 3Methylperylene does not have any functional group that can engage in hydrogen bonding with the host, but the presence of the methyl group will let us know the effect of a non-polar substituent on the periphery of the guest molecule. If only the steric influence is at play, then we should expect a decrease of the association constant with respect to that shown when unfunctionalized perylene was used. If, on the contrary, the binding affinity is increased, then we should consider that other type of non-covalent interactions might be playing a role in the stabilization of the resulting host-guest complex. As we did for the other guests used in this work, we performed the corresponding ${ }^{1} \mathrm{H} \mathrm{NMR}$ titration in $\mathrm{CD}_{2} \mathrm{Cl}_{2}$ at room temperature. The titration with 6 produced similar perturbations of the signals of the protons of the host, as those shown for the titrations with 3-5, although in this case the maximum downfield shift shown for the signal due to the $\mathrm{NH}$ proton was $0.39 \mathrm{ppm}$, therefore significantly smaller than those observed during the titrations with $\mathbf{4}$ and $\mathbf{5}$. As happened for the titration with 5, the maximum shifts were attained when a 1:1 (host:guest) stoichiometry was achieved, therefore indicating that the association constant was very high and exceeded the limits for its determination by ${ }^{1} \mathrm{H}$ NMR spectroscopy. This made us proceed as we did before for the case of 3-perylenemethanol. In this case, we performed an experiment in which we added one equivalent of 6 to a pre-formed sample of perylene@1. The ${ }^{1} \mathrm{H}$ NMR spectrum of the sample indicated that the molecule of $\mathbf{6}$ had been trapped in the cavity of 1 forming 6@1, with the concomitant release of perylene (see ESI for full details). This experiment indicates that the association constant for the formation of $6 @ 1$ is larger than that for perylene@1. In a similar manner, we 
wanted to know if the binding affinity of 6 with the metallorectangle could be compared with the large affinity shown by $\mathbf{5}$. By performing a similar competitive experiment, this time adding 5 on a pre-formed sample of 6@1, we observed that the binding constant associated to the formation of $\mathbf{5} @ 1$ is larger than that for 6@1. Therefore, the order of the association constants was established as perylene@1<6@1<5@1, with the association constant of perylene@1 being 1.6×105 $\mathrm{M}^{-1}$, as we previously published. . $^{9 a}$

To add to the experimental data, we optimized the structures and obtained the energies of inclusion complexes of perylene, 3-metyl-perylene (6), 3ethylperylene (7), and 3-perylenemethanol (5) with a truncated model of 1, using the MN15-L/def2-QZVP DFT method in a chloroform solvent continuum. The truncated host model $\mathbf{1}^{\prime}$ differed from 1 by the replacement of NBu groups by NMe, and by replacement of the remote $t$-Bu groups of the carbazole fragments by hydrogen atoms. This model 1' retained the important four $t$-Bu groups on the pyrene fragments. The computed geometries differed slightly. Perylene was nearly perfectly centered in perylene@1', the orientation reflected in the $\mathrm{X} 1-\mathrm{X} 2-\mathrm{X} 3$ angle being $179.5^{\circ}$ where $\mathrm{X} 2$ is the centroid of the perylene, and $X 1$ and $X 3$ are the centroids of the pyrene fragments of $1 '$. The calculated X1-X3 distance, $6.97 \AA$, was similar to the experimental in 5@1, 7.14 A. The calculated structures of 6@1' and 7@1' displayed a slight shift of the guest in the cavity, resulting in the X1-X2-X3 angles of 177.1 and $172.9^{\circ}$, respectively. This slight horizontal shift was apparently to avoid the Me and Et substitutes approaching the nearby gold atoms too closely. As a result, the carbazole N-X2 distances became unequal, e.g., 10.85 and $10.75 \AA$ in 7@1'. The X1-X3 distances were slightly shorter in 6@1' and 7@1': 6.88 and $6.92 \AA$, respectively, compared to perylene@1'. Finally, the calculated structure of 5@1' was characterized by the $\mathrm{X} 1-\mathrm{X} 2-\mathrm{X} 3$

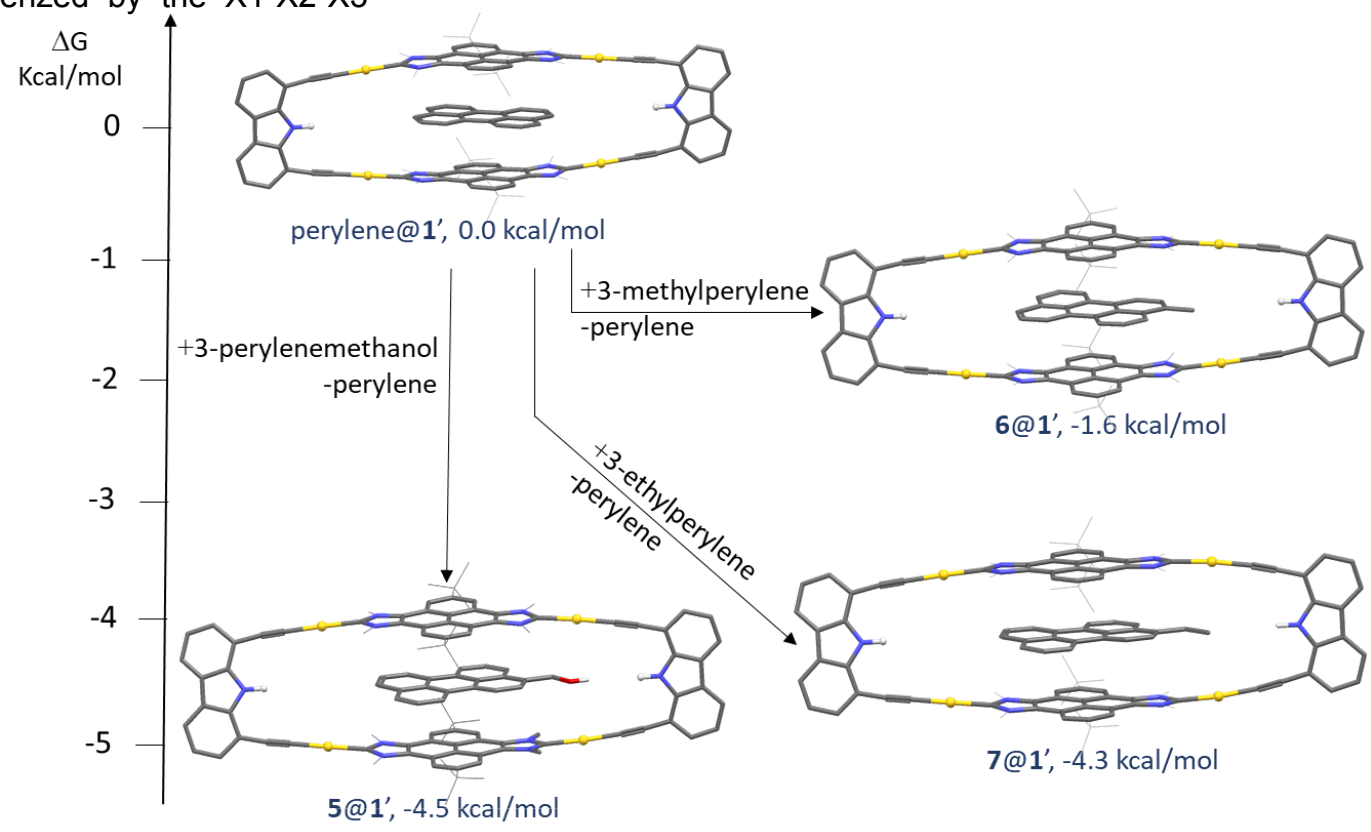


Figure 3. Calculated host-guest structures of 1' with perylene, 3-methylperylene, 3-ethylperylene and 3-perylenemethanol. The energy diagram represents the calculated Gibbs energies for the substitution of perylene by 3-perylenemethanol (5), 3methylperylene (6), and 3-ethylperylene (7).

angle of $178.9^{\circ}$, and the $\mathrm{X} 1-\mathrm{X} 3$ separation of $6.93 \AA$. The calculated $\mathrm{NH} \cdots \mathrm{OH}$ separation of $3.69 \AA$ was too long for a hydrogen bonding interaction.

Using the calculated molar Gibbs energies of the four inclusion complexes, we derived the reaction energies for substitution of perylene in perylene@1' by 5,6 , and 7. These reactions were increasingly exergonic in the order $-1.6(6)>-4.3(7)>-4.5 \mathrm{kcal} / \mathrm{mol}(5)$, in a qualitative agreement with the experimental data for 5 and 6 (Figure 3). It is of note the more favorable reaction energy with 7 vs $\mathbf{6}$ and similar reaction energies of 5 and 7 , suggesting that dispersive interactions make an important contribution to the guest-host bonding energy. The dispersive interactions are expected to correlate with the size or surface area of the host.

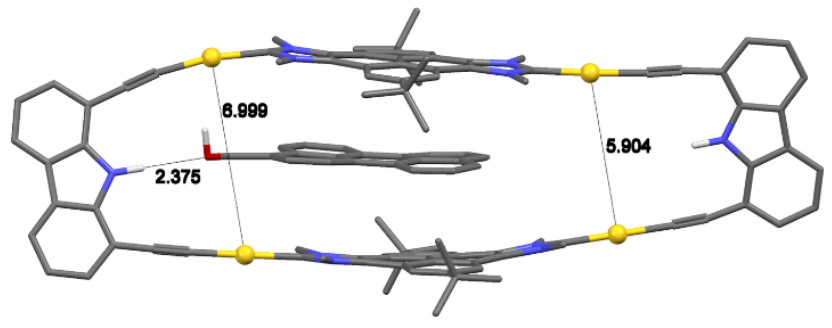

Figure 4. Calculated host-guest structure of $\mathrm{H}$-bonded 5@1'. Distances are given in Å.

Finally, an attempt was made to calculate a structure of 5@1' with 3-perylenemethanol shifted inside the cage enough to establish a hydrogen bond with an $\mathrm{NH}$ group of 1' (Figure 4). Indeed, such structure could be optimized, characterized by the $\mathrm{X} 1-\mathrm{X} 2-\mathrm{X} 3$ angle of $137.2^{\circ}, \mathrm{X} 1-\mathrm{X} 3$ distance of $7.02 \AA$, and the $\mathrm{NH} \cdots \mathrm{OH}$ distance of $2.375 \AA$ consistent with a weak hydrogen bond. This product structure was $4.0 \mathrm{kcal} / \mathrm{mol}$ higher in energy compared to the structure of 5@1' described above, indicating that the energy of hydrogen bonding is not sufficient to induce the horizontal shift of the guest in $1^{\prime}$. In addition, as can be observed in Figure 4, the structure of the host is highly distorted when compared with the X-ray diffraction structure of free $1,{ }^{9 e}$ and also with respect to the DFT calculated structures shown in Figure 3. The two gold atoms close to the $\mathrm{NH}$ group where the hydrogen bonding interaction is taking place are separated by a distance of about $7 \AA$, while the separation between the other two gold atoms is of $5.9 \AA$. The larger separation of the two gold atoms close to the hydrogen bonded $\mathrm{NH}$ group facilitates the accommodation of the guest, with the oxygen located between the two gold atoms at a distance of 3.30-3.33 $\AA$ (the sum of the van der Waals radii for $\mathrm{Au}$ and $\mathrm{O}$ is $3.18 \AA$ ). However, the structural distortion produced in the host has an energy cost that may also help to explain the higher energy of the structure depicted in Figure 4 compared to the non-hydrogen bonded structure of 5@1' shown in Figure 3.

\section{CONCLUSIONS}

In summary, we performed a study to determine the influence of substituents on PAHs on the binding affinities of these molecules with a tetra-Au(I)-based metallorectangle 1. We expected that the presence of a carbazole linker in the host could encourage 2naphthalenol, 1-pyrenemethanol, or 3-perylenemethanol to form a hydrogen bond with one of the $\mathrm{NH}$ groups of the host and, thus, might lead to an enhanced binding affinity due to the combined $\pi-\pi$ stacking and hydrogenbonding interactions. Our results indicate that, for the smaller PAHs (anthracene and pyrene), the added alcohol group does not produce any enhancement in the binding affinity with 1 , while for perylene, the addition of a $\mathrm{CH}_{2} \mathrm{OH}$ group produced a significant increase of the binding affinity of the guest compared to that of the parent perylene. We further observed that the methyl group of 3-methylpyrene also produced an enhancement of the binding affinity of the guest, compared to perylene. As confirmed by X-ray crystallography and by DFT calculations, the host-guest complex 3pyrenemethanol@1 does not show hydrogen bonding between the guest and the host. The $\mathrm{NH}$ group is, in principle, capable of hydrogen bonding to the $\mathrm{OH}$ of the guest, but the calculated energy of the corresponding DFT-optimized hydrogen-bonded structure is 4.0 $\mathrm{kcal} / \mathrm{mol}$ larger than the structure not possessing a hydrogen bond. This is because to form the hydrogen bond, the guest must shift from the position of the best face-to-face surface overlap with 1 , needed for optimum $\pi-\pi$-stacking. This would also produce an important distortion of the structure of the molecule, which has an energy cost. On the other hand, the introduction of a methyl or an ethyl group at the periphery of the perylene molecule produces a stabilization of the resulting hostguest inclusion complex, compared to the one with encapsulated perylene. For the system under study, it seems clear that London dispersion can make a significant contribution to the host-guest bonding energy (on the order of $5 \mathrm{kcal} / \mathrm{mol}$ ). Therefore, our work highlights the importance of dispersive interactions in the contribution to the binding affinity of host-guest chemistry complexes. The role of this type of non-covalent interaction in supramolecular complexation processes is still widely debated ${ }^{13}$ and, only recently, a few studies on how dispersive interactions influence the binding affinities in host-guest systems have been reported. ${ }^{11,14}$

\section{ACKNOWLEDGEMENTS}

We gratefully acknowledge financial support from the Ministerio de Ciencia y Universidades (PGC2018093382-B-I00) and the Universitat Jaume I (UJI-B201707 and UJI-A2017-02). We are grateful to the Serveis Centrals d'Instrumentació Científica (SCIC-UJI) for providing with spectroscopic facilities.

\section{ASSOCIATED CONTENT}

\section{Supporting Information}


All experimental details, including NMR, Uv-vis and fluorescence spectra, titration experiments, X-ray diffraction and computational details.

The Supporting Information is available free of charge on the ACS Publications website.

\section{AUTHOR INFORMATION}

\section{Corresponding Author}

*Eduardo Peris: eperis@uji.es

\section{Notes}

The authors declare no competing financial interests.

\section{REFERENCES}

(1) a) Keyte, I. J.; Harrison, R. M.; Lammel, G. Chemical reactivity and long-range transport potential of polycyclic aromatic hydrocarbons - a review. Chem. Soc. Rev. 2013, 42, 9333-9391; b) Haritash, A. K.; Kaushik, C. P. Biodegradation aspects of Polycyclic Aromatic Hydrocarbons (PAHs): A review. J. Hazard. Mater. 2009, 169, 1-15; c) Lemieux, C. L.; Lambert, A. B.; Lundstedt, S.; Tysklind, M.; White, P. A. Mutagenic hazards of complex polycyclic aromatic hydrocarbon mixtures in contaminated soil. Environ. Toxicol. Chem. 2008, 27, 978-990; d) Srogi, $\mathrm{K}$. Monitoring of environmental exposure to polycyclic aromatic hydrocarbons: a review. Environmental Chemistry Letters 2007, 5, 169-195.

(2) a) Narita, A.; Wang, X. Y.; Feng, X. L.; Mullen, K. New advances in nanographene chemistry. Chem. Soc. Rev. 2015, 44, 6616-6643; b) Origuchi, S.; Kishimoto, M.; Yoshizawa, M.; Yoshimoto, S. A Supramolecular Approach to the Preparation of Nanographene Adlayers Using WaterSoluble Molecular Capsules. Angew. Chem. Int. Ed. 2018, 57, 15481-15485; c) Figueira-Duarte, T. M.; Mullen, K. Pyrene-Based Materials for Organic Electronics. Chem. Rev. 2011, 111, 7260-7314; d) Wu, J.; Pisula, W.; Muellen, K. Graphenes as potential material for electronics. Chem. Rev. 2007, 107, 718-747; e) Feng, X.; Hu, J. Y.; Redshaw, C.; Yamato, T. Functionalization of Pyrene To Prepare Luminescent Materials-Typical Examples of Synthetic Methodology. Chem. Eur. J. 2016, 22, 11898-11916; f) Metzger, R. M. Unimolecular Electronics. Chem. Rev. 2015, 115, 5056-5115; g) Guo, X.; Baumgarten, M.; Mullen, K. Designing pi-conjugated polymers for organic electronics. Prog. Polym. Sci. 2013, 38, 1832-1908; h) Mullen, K.; Rabe, J. P. Nanographenes as active components of single-molecule electronics and how a scanning tunneling microscope puts them to work. Acc. Chem. Res. 2008, 41, 511-520; i) Wu, J. S.; Pisula, W.; Mullen, K. Graphenes as potential material for electronics. Chem. Rev. 2007, 107, 718-747.

(3) a) Mazzeo, M.; Vitale, V.; Della Sala, F.; Anni, M.; Barbarella, G.; Favaretto, L.; Sotgiu, G.; Cingolani, R.; Gigli, G. Bright white organic light-emitting devices from a single active molecular material. Adv. Mater. 2005, 17, 34-39; b) Barbarella, G.; Favaretto, L.; Sotgiu, G.; Zambianchi, M.; Fattori, V.; Cocchi, M.; Cacialli, F.; Gigli, G.; Cingolani, R. Modified oligothiophenes with high photo - and electroluminescence efficiencies. Adv. Mater. 1999, 11, 1375-1379.

(4) a) Li, X. C.; Sirringhaus, H.; Garnier, F.; Holmes, A. B.; Moratti, S. C.; Feeder, N.; Clegg, W.; Teat, S. J.; Friend, R. $\mathrm{H}$. A highly pi-stacked organic semiconductor for thin film transistors based on fused thiophenes. J. Am. Chem. Soc. 1998, 120, 2206-2207; b) Laquindanum, J. G.; Katz, H. E.; Lovinger, A. J.; Dodabalapur, A. Benzodithiophene rings as semiconductor building blocks. Adv. Mater. 1997, 9, 36-39.
(5) Nagamoto, Y.; Yamaoka, Y.; Fujimura, S.; Takemoto, Y.; Takasu, K. Synthesis of Functionalized Polycyclic Aromatic Compounds via a Formal 2+2 -Cycloaddition. Org. Lett. 2014, 16, 1008-1011.

(6) a) Zhang, D. W.; Ronson, T. K.; Lavendomme, R.; Nitschke, J. R. Selective Separation of Polyaromatic Hydrocarbons by Phase Transfer of Coordination Cages. J. Am. Chem. Soc. 2019, 141, 18949-18953; b) Wu, G. C.; Wang, C. Y.; Jiao, T. Y.; Zhu, H. T. Z.; Huang, F. H.; Li, H. Controllable SelfAssembly of Macrocycles in Water for Isolating Aromatic Hydrocarbon Isomers. J. Am. Chem. Soc. 2018, 140, 59555961; c) Alvarino, C.; Pia, E.; Garcia, M. D.; Blanco, V.; Fernandez, A.; Peinador, C.; Quintela, J. M. Dimensional Matching of Polycyclic Aromatics with Rectangular Metallacycles: Insertion Modes Determined by $\mathrm{C}-\mathrm{H}$ center dot center dot center dot pi Interactions. Chem. Eur. J. 2013, 19, 15329-15335; d) Peinador, C.; Pia, E.; Blanco, V.; Garcia, M. D.; Quintela, J. M. Complexation of Pyrene in Aqueous Solution with a Self-Assembled Palladium Metallocycle. Org. Lett. 2010, 12, 1380-1383; e) Blanco, V.; Garcia, M. D.; Terenzi, A.; Pia, E.; Fernandez-Mato, A.; Peinador, C.; Quintela, J. M. Complexation and Extraction of PAHs to the Aqueous Phase with a Dinuclear Pt-II Diazapyrenium-Based Metallacycle. Chem. Eur. J. 2010, 16, 12373-12380; f) Jiao, T. Y.; Cai, K.; Liu, Z. C.; Wu, G. C.; Shen, L. B.; Cheng, C. Y.; Feng, Y. N.; Stern, C. L.; Stoddart, J. F.; Li, H. Guest recognition enhanced by lateral interactions. Chem. Sci. 2019, 10, 5114-5123; g) Dale, E. J.; Vermeulen, N. A.; Thomas, A. A.; Barnes, J. C.; Juricek, M.; Blackburn, A. K.; Strutt, N. L.; Sarjeant, A. A.; Stern, C. L.; Denmark, S. E.; Stoddart, J. F. ExCage. J. Am. Chem. Soc. 2014, 136, 10669-10682; h) Juricek, M.; Barnes, J. C.; Dale, E. J.; Liu, W. G.; Strutt, N. L.; Bruns, C. J.; Vermeulen, N. A.; Ghooray, K. C.; Sarjeant, A. A.; Stern, C. L.; Botros, Y. Y.; Goddard, W. A.; Stoddart, J. F. Ex(2)Box: Interdependent Modes of Binding in a Two-NanometerLong Synthetic Receptor. J. Am. Chem. Soc. 2013, 135, 12736-12746; i) Barnes, J. C.; Juricek, M.; Strutt, N. L.; Frasconi, M.; Sampath, S.; Giesener, M. A.; McGrier, P. L.; Bruns, C. J.; Stern, C. L.; Sarjeant, A. A.; Stoddart, J. F. ExBox: A Polycyclic Aromatic Hydrocarbon Scavenger. J. Am. Chem. Soc. 2013, 135, 183-192; j) Samanta, J.; Natarajan, R. Cofacial Organic Click Cage to Intercalate Polycyclic Aromatic Hydrocarbons. Org. Lett. 2016, 18, 3394-3397.

(7) a) Pöthig, A.; Casini, A. Recent Developments of Supramolecular Metal-based Structures for Applications in Cancer Therapy and Imaging. Theranostics 2019, 9, 31503169; b) Gan, M. M.; Liu, J. Q.; Zhan, L.; Wang, Y. Y.; Hahn, F. E.; Han, Y. F. Preparation and Post-Assembly Modification of Metallosupramolecular Assemblies from Poly(N-Heterocyclic Carbene) Ligands. Chem. Rev. 2018, 118, 9587-9641; c) Sinha, N.; Hahn, F. E. Metallosupramolecular Architectures Obtained from Poly-Nheterocyclic Carbene Ligands. Acc. Chem. Res. 2017, 50, 2167-2184; d) Li, Y.; An, Y. Y.; Fan, J. Z.; Liu, X. X.; Li, X.; Hahn, F. E.; Wang, Y. Y.; Han, Y. F. Strategy for the Construction of Diverse Poly-NHC-Derived Assemblies and Their Photoinduced Transformations. Angew. Chem. Int. Ed. 2020, 59, 10073-10080; e) Wang, Y. S.; Feng, T.; Wang, Y. Y.; Hahn, F. E.; Han, Y. F. Homo- and Heteroligand Poly-NHC Metal Assemblies: Synthesis by Narcissistic and Social Self-Sorting. Angew. Chem. Int. Ed. 2018, 57, 15767-15771; f) Fan, Q. J.; Lin, Y. J.; Hahn, F. E.; Jin, G. X. Host-guest capability of a three-dimensional heterometallic macrocycle. Dalton Trans. 2018, 47, 22402246; g) Sinha, N.; Roelfes, F.; Hepp, A.; Mejuto, C.; Peris, E.; Hahn, F. E. Synthesis of Nanometer-Sized Cylinder-Like Structures from a 1,3,5-Triphenylbenzene-Bridged TrisNHC Ligand and Ag-I, Au-I, and Cu-I. Organometallics 2014, 33, 6898-6904; h) Segarra, C.; Guisado-Barrios, G.; 
Hahn, F. E.; Peris, E. Hexanuclear cylinder-shaped assemblies of silver and gold from benzene-hexa-Nheterocyclic carbenes. Organometallics 2014, 33, 50775080; i) Weger, M.; Grotsch, R. K.; Knaus, M. G.; Giuman, M. M.; Mayer, D. C.; Altmann, P. J.; Mossou, E.; Dittrich, B.; Pöthig, A.; Rieger, B. Non-Innocent Methylene Linker in Bridged Lewis Pair Initiators. Angew. Chem. Int. Ed. 2019, 58, 9797-9801; j) Altmann, P. J.; Pöthig, A. Pillarplexes: A Metal-Organic Class of Supramolecular Hosts. J. Am. Chem. Soc. 2016, 138, 13171-13174; k) Zhang, Y. W.; Bai, S.; Wang, Y. Y.; Han, Y. F. A Strategy for the Construction of Triply Interlocked Organometallic Cages by Rational Design of Poly-NHC Precursors. J. Am. Chem. Soc. 2020, 142, 13614-13621; I) Li, Y.; Jin, G. F.; An, Y. Y.; Das, R.; Han, Y. F. Metal-Carbene-Templated Photochemistry in Solution: A Universal Route towards Cyclobutane Derivatives. Chin. J. Chem . 2019, 37, 1147-1152; m) Ma, L. L.; An, Y. Y.; Sun, L. Y.; Wang, Y. Y.; Hahn, F. E.; Han, Y. F. Supramolecular Control of Photocycloadditions in Solution: In Situ Stereoselective Synthesis and Release of Cyclobutanes. Angew. Chem. Int. Ed. 2019, 58, 3986-3991.

(8) Ibáñez, S.; Poyatos, M.; Peris, E. N-Heterocyclic carbenes: a door open to supramolecular organometallic chemistry. Acc. Chem. Res. 2020, 53, 1401-1413.

(9) a) Ibanez, S.; Peris, E. Dimensional Matching versus Induced-Fit Distortions: Binding Affinities of Planar and Curved Polyaromatic Hydrocarbons with a Tetragold Metallorectangle. Angew. Chem. Int. Ed. 2020, 59, 68606865 ; b) Martinez-Agramunt, V.; Peris, E. A palladiumhinged organometallic square with a perfect-sized cavity for the encapsulation of three heteroguests. Chem. Commun. 2019, 55, 14972-14975; c) Ibáñez, S.; Peris, E. A Rigid Trigonal-Prismatic Hexagold Metallocage That Behaves as a Coronene Trap. Angew. Chem. Int. Ed. 2019, 58, 66936697; d) Martinez-Agramunt, V.; Ruiz-Botella, S.; Peris, E. Nickel-Cornered Molecular Rectangles as Polycyclic Aromatic Hydrocarbon Receptors. Chem. Eur. J. 2017, 23, 6675-6681; e) Biz, C.; Ibáñez, S.; Poyatos, M.; Gusev, D.; Peris, E. Gold(I) Metallo-Tweezers for the Recognition of Functionalized Polycyclic Aromatic Hydrocarbons by Combined pi-pi Stacking and H-Bonding. Chem. Eur. J. 2017, 23, 14439-14444.

(10) Liptrot, D. J.; Power, P. P. London dispersion forces in sterically crowded inorganic and organometallic molecules. Nature Reviews Chemistry 2017, 1.

(11) Haberhauer, G.; Woitschetzki, S.; Bandmann, H. Strongly underestimated dispersion energy in cryptophanes and their complexes. Nat. Commun. 2014, 5.

(12) a) Lowe, A. J.; Pfeffer, F. M.; Thordarson, P. Determining binding constants from $\mathrm{H}-1$ NMR titration data using global and local methods: a case study using $n$ polynorbornanebased anion hosts. Supramol. Chem. 2012, 24, 585-594; b) Thordarson, P. Determining association constants from titration experiments in supramolecular chemistry. Chem. Soc. Rev. 2011, 40, 1305-1323.

(13) Hunter, C. A. van der Waals interactions in non-polar liquids. Chem. Sci. 2013, 4, 834-848.

(14) a) Melix, P.; Heine, T. London Dispersion Governs the Interaction Mechanism of Small Polar and Nonpolar Molecules in Metal-Organic Frameworks. J. Phys. Chem. C 2020, 124, 11985-11989; b) Biedermann, F.; Schneider, H. J. Experimental Binding Energies in Supramolecular Complexes. Chem. Rev. 2016, 116, 5216-5300; c) Schneider, H. J. Dispersive Interactions in Solution Complexes. Acc. Chem. Res. 2015, 48, 1815-1822. 
Table of Contents artwork

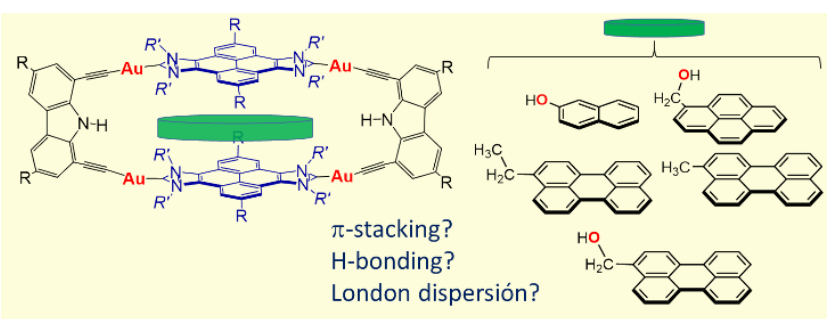

